\title{
Pricing and Hedging Double-Barrier Options: A Probabilistic Approach*
}

\author{
Hélyette Geman ${ }^{1)}$ and Marc Yor ${ }^{2)}$
}

\begin{abstract}
Barrier options have become increasingly popular over the last few years. Less expensive than standard options, they may provide the appropriate hedge in a number of risk management strategies. In the case of a single barrier option, the valuation problem is not very difficult (see Merton 193, Goldman-Sosin-Gatto 1979). The situation where the option gets knocked out when the underlying instrument hits either of two well-defined boundaries is less straightforward. Kunitomo and Ikeda (1992) provide a pricing formula expressed as the sum of an infinite series whose convergence is studied through numerical procedures and suggested to be rapid. We follow a methodology which proved quite successful in the case of Asian options (see Geman-Yor 1992, 1993) and which has its roots in some fundamental properties of Brownian motion. This methodology permits the derivation of a simple expression of the Laplace transform of the double-barrier price with respect to its maturity date. The inversion of the Laplace transform is then fairly easy to perform, using the techniques developed by Geman-Eydeland (1995).
\end{abstract}

\section{Keywords}

Double-barrier option, geometric Brownian motion, Cameron-Martin theorem, Laplace transform inversion.

1) University of Reims \& ESSEC, Finance Department, Avenue Bernard Hirsch, B. P. 105, 95021 Cergy-Pontoise Cedex (France); Tel: + 33-1-34433000, Fax: + 33-1-34433001.

2) University Paris VI, Laboratoire de Probabilités. 


\section{INTRODUCTION}

Barrier options have become increasingly popular over the last few years. Less expensive than the equivalent standard options, they may be the appropriate hedge in a number of situations. For instance, a down-and-in put with a low barrier offers an inexpensive protection against a big downward move of the underlying asset ; in the same manner, an up-andout call on an index may enhance at a cheap cost an already existing long position in the index.

The pricing of "single barrier" options is not difficult in the standard Black and Scholes framework. An arbitrage approach leads to a fairly elementary mathematical problem and closed-form solutions have been available for some time. For instance, in his seminal paper of 1973, Merton presented a pricing formula for an option whose pay-off is restricted by a floor knock-out boundary ; in 1979, Goldman, Sosin and Gatto offered closed-form solutions for all types of barrier options. The problem of double-barrier options which we address in this article is more difficult and, to our knowledge, no simple formula currently exists. Consequently, practitioners who trade these instruments rely heavily on the use of simulations to obtain option prices. Simulation is indeed an effective and simple tool readily that is applicable to the problem of valuing some of the most complex contracts. However, Fu, Madan and Wang (1995) show that for continuous time Asian options, and suspect that for other continuous time path-dependent options as well, naive simulations based on pricing strategies are expensive, inefficient and inaccurate. They even find that decreasing the standard error with more replications only serves to increase the confidence in the wrong valuation. Lastly, Geman and Eydeland (1995) show the remarkable superiority of an exact approach over Monte-Carlo simulations when delta hedging of Asian options is at stake.

Our goal is to adopt the same type of "exact" pricing methodology in the case of double-barrier options as the one we followed for Asian options. We use the standard risk-neutral valuation techniques and the Markov property as well as the Cameron-Martin theorem to obtain a simple expression of the Laplace transform of the option price. As it is the case for the double-barrier options traded in the markets, we will only consider the situation of fixed boundaries. 


\section{PRICING DOUBLE-BARRIER OPTIONS}

A double-knock option is characterized by two barriers L (lower barrier) and $\mathrm{U}$ (upper barrier) ; the option knocks out if either barrier is touched. Otherwise, the option gives at maturity $\mathrm{T}$ the standard Black and Scholes pay-off $\max (0, S(T)-k)$, where $k$, the strike price of the option, satisfies $\mathrm{L}<\mathrm{k}<\mathrm{U}$.

The distribution of the range of Brownian motion was studied as early as 1941 by Bachelier while the trivariate joint distribution of $\left(I_{t}=\inf _{u t} W_{u}, L_{t}=\sup _{u s t} W_{u}, W_{t}\right)$ has been known for some time (see e.g. Freedman (1971) or Revuz-Yor (1994, p.106)) and is expressed as

$$
\begin{aligned}
& P\left(a \leq I_{t} \leq L_{t} \leq b ; W_{t} \in d x\right) \\
& =(2 \pi t)^{-\frac{1}{2}} \sum_{k=-\infty}^{\infty}\left\{\exp \left(-\frac{1}{2 t}(x+2 k(b-a))^{2}\right)-\exp \left(-\frac{1}{2 t}((x-2 b)+2 k(b-a))^{2}\right)\right\} d x
\end{aligned}
$$

This result and variants of it are found in many places in the probabilistic literature (see e.g., Doob (1949), Feller (1970), Shorack-Wellner (1986), Teunen-Goovaerts (1994)). It is this path which is followed by Kunitomo and Ikeda (1992) who propose pricing formulas expressed as theta function type series for European options whose pay-off is restricted by general curve boundaries.

In our setting, the uncertainty in the economy is represented by a filtered probability space $\left(\Omega, \mathfrak{H}_{1}, \mathfrak{H}_{t}, P\right)$, where $\mathfrak{H}_{t}$ is the information available at time $t$ and $P$ is the objective probability. From the seminal papers by Harrison-Kreps (1979) and Harrison-Pliska (1981) we know that the noarbitrage assumption implies the existence of a probability measure $Q$ equivalent to $P$ such that the discounted prices of the basic securities are $Q$ martingales. Under the probability $Q$, the dynamics of the price $S(t)$ of the underlying asset are driven by the stochastic differential equation

$$
\frac{d S_{t}}{S_{t}}=y d t+\sigma d \tilde{W}_{t}
$$


where $(\tilde{W}(t))$ is a Q-Brownian motion, $\sigma$ is constant, and the risk-neutral drift is assumed to be a general constant. So, we can incorporate the case where the underlying instrument is a dividend-paying stock, a currency or a commodity. Equation (1) immediately gives

$$
S(t)=S(0) \exp \left\{\left(y-\frac{\sigma^{2}}{2}\right) t+\sigma \tilde{W}(t)\right\}
$$

The call price is equal to

$$
C_{L, U}(t)=e^{-r(T-t)} E_{Q}\left[(S(T)-k)^{+} 1_{\left(\Sigma_{L, U}>T\right)} / J_{t}\right]
$$

where $\Sigma_{L, U}$ is the first exit time of the process $\left(S_{t}\right)_{t \geq 0}$ out of the interval [L,U]. We can write for all $u \geq t \geq 0$

$$
S(u)=S(t) S_{1}(u-t)
$$

where $S_{1}(s)=\exp \left\{\left(y-\frac{\sigma^{2}}{2}\right) s+\sigma \hat{W}(s)\right\}$ and $(\hat{W}(s)=\tilde{W}(t+s)-\tilde{W}(t), s \geq 0)$ is a new Brownian motion under $Q$. Denoting by $\hat{\Sigma}_{\alpha, \beta}$ the first exit time of the process $\left(S_{1}(s)\right)_{s \geq 0}$ out of the interval $[\alpha, \beta]$, we obtain the immediate equality

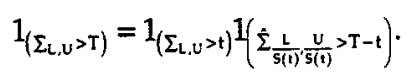

Since the variable $1_{\left(\Sigma_{L, U}>t\right)}$ is $\mathfrak{I}_{t}$ measurable, we can simplify for all $t<T$

$$
\begin{aligned}
& E_{Q}\left[(S(T)-k)^{+} 1_{\left(\Sigma_{L, U}>T\right)} / \mathbb{J}_{t}\right] \\
& =1\left(\Sigma_{L, U}>t\right) E_{Q}\left[\left(S(t) S_{1}(T-t)-k\right)^{+} 1\left(\hat{\Sigma} \frac{L}{S(t)}, \frac{U}{S(t)}>T-t\right) / \mathbb{I}_{t}\right] .
\end{aligned}
$$

Consequently, if the double-barrier call has not been knocked out prior to time $t$, its price at time $t$ is equal to

$$
C_{L, U}(t)=e^{-r(T-t)} S(t) E_{Q}\left[\left(S_{1}(\tau)-h\right)^{+} 1_{\left(\hat{\Sigma}_{m, M}>\tau\right)}\right],
$$


where $\tau=T-t, h=\frac{k}{S(t)}, m=\frac{L}{S(t)}$ and $M=\frac{U}{S(t)}$.

We now observe that, rather than computing the expectation in (3) it is easier to compute the quantity

$$
\varphi_{m, M}^{(h)}(\tau)=E_{Q}\left[\left(S_{1}(\tau)-h\right)^{+} 1_{\left(\hat{\Sigma}_{m, M}<\tau\right)}\right] .
$$

This will give, by difference with the Black and Scholes formula and an appropriate adjustment, the double-knock call price as

$$
C_{L, U}(t)=S(t) B S(0,1, \sigma, h, \tau)-e^{-\pi} S(t) \varphi_{m, M}^{(h)}(\tau)
$$

where $\mathrm{BS}(0,1, \sigma, h, \tau)$ denotes the price at time 0 of a standard call with maturity $\tau$ and strike price $h$ written on an underlying stock which has value 1 at time 0 and volatility $\sigma$.

The quantity $\varphi_{m, M}^{(h)}(\tau)$ has a fairly simple expression when $\tau$ is replaced by an exponential variable independent of $S_{1}$ (the reader interested in this question can find in Appendix 2 a general discussion of the issue : why is it easier to handle a functional of a Brownian motion $W$ at an independent exponential time rather than at a fixed time ?). This leads us to consider in this specific case the following Laplace transform with respect to time :

$$
\psi(\lambda)=\int_{0}^{+\infty} \mathrm{e}^{-\lambda \tau} \varphi_{\mathrm{m}, M}^{(h)}(\tau) \mathrm{d} \tau
$$

Making the change of variables $u=\tau \sigma^{2}$, the right-hand side of (5) becomes

$$
\int_{0}^{\infty} \frac{\mathrm{du}}{\sigma^{2}} \mathrm{e}^{-\lambda \mathbf{u} / \sigma^{2}} \varphi_{m, M}^{(\mathbf{h})}\left(\frac{\mathrm{u}}{\sigma^{2}}\right)
$$

where, denoting $\hat{v}=y-\frac{\sigma^{2}}{2}$ and $W(u)=\sigma \hat{W}\left(\frac{u}{\sigma^{2}}\right)$, we obtain

$$
\left.\varphi_{m, M}^{(h)}\left(\frac{u}{\sigma^{2}}\right)=E\left[\left(\exp \left(W(u)+\hat{v} \frac{u}{\sigma^{2}}\right)-h\right)^{+} 1_{\hat{\mathcal{I}}_{m, M}<\frac{u}{\sigma^{2}}}\right)\right] .
$$


Recalling the definition of

$$
\hat{\Sigma}_{m, M}=\inf \{s: \exp (\sigma \hat{W}(s)+\hat{v} s)=m \text { or } M\},
$$

we can write equivalently

$$
\begin{aligned}
\sigma^{2} \hat{\Sigma}_{m, M} & =\inf \left\{x: \exp \left(\sigma \hat{W}\left(\frac{x}{\sigma^{2}}\right)+\frac{\hat{v} x}{\sigma^{2}}\right)=m \text { or } M\right\} \\
& =\inf \left\{x: \exp \left(W(x)+\frac{\hat{v} x}{\sigma^{2}}\right)=m \text { or } M\right\},
\end{aligned}
$$

where $(W(x))$ was introduced earlier.

$$
\begin{aligned}
& \text { We define } v=\frac{\hat{v}}{\sigma^{2}}=\frac{1}{\sigma^{2}}\left(y-\frac{\sigma^{2}}{2}\right) \text { and introduce } \\
& \Sigma_{m, M}^{(v)}=\sigma^{2} \hat{\Sigma}_{m, M}
\end{aligned}
$$

where $\Sigma_{m, M}^{(v)}$ represents the first time $m$ or $M$ is reached by the process $X_{t}^{(v)} \equiv \exp (W(t)+v t)$. Hence,

$$
\varphi_{m, M}^{(h)}\left(\frac{u}{\sigma^{2}}\right)^{\operatorname{def}}=\varphi(u) \equiv E\left[\left\{X_{u}^{(v)}-h\right\}^{+} 1\left(\sum_{m, M}^{(v)}<u\right)\right]
$$

and

$$
\psi(\lambda)=\int_{0}^{\infty} \frac{d u}{\sigma^{2}} e^{\frac{-\lambda u}{\sigma^{2}}} \varphi(u)=\frac{1}{\sigma^{2}} \Phi\left(\frac{\lambda}{\sigma^{2}}\right)
$$

where the function $\Phi$ is defined as the Laplace transform of $\varphi$, i.e.,

$$
\Phi(\theta)=\int_{0}^{\infty} d u e^{-\theta u} \varphi(u)=E\left[\int_{\sum_{m, M}^{(v)}}^{\infty} e^{-\theta u}\left(X_{u}^{(v)}-h\right)^{+} d u\right] .
$$

From now on, we will focus on the computation of $\Phi(\theta)$. Let us denote for simplicity $f(x)=(x-h)^{+}$and $\Sigma=\Sigma_{m, M}^{(v)}$ and introduce the positive numbers a 
and $b$ such that $m=e^{-a}, M=e^{b}$. By using the strong Markov property, we simplify the expression of $\Phi(\theta)$

$$
\Phi(\theta)=E\left[e^{-\theta \Sigma} E_{X_{\Sigma}^{(v)}}\left(\int_{0}^{\infty} e^{-\theta u} f\left(X_{u}^{(v)}\right)\right) d u\right]=E\left[e^{-\theta \Sigma}\left(V_{\theta}^{(v)} f\right)\left(X_{\Sigma}^{(v)}\right)\right],
$$

where

$$
V_{\theta}^{(v)} f(x)=E_{x}^{v}\left[\int_{0}^{\infty} e^{-\theta u} f\left(X_{u}\right) d u\right]
$$

and $E_{x}^{v}$ is the expectation relative to $P_{x}^{v}$, where $P_{x}^{v}$ is the law of the process $\left(X_{u}^{(v)}, u \geq 0\right)$ originating in $x$. To avoid confusion between Brownian and geometric Brownian motions, we use $\hat{\mathbf{E}}_{z}$ for the expectation with respect to $\hat{\mathrm{P}}_{z}$, the Wiener measure of Brownian motion starting at $z$.

Denoting $g=V_{\theta}^{(v)} f$, and using the Cameron-Martin absolute continuity relationship between $\left(W_{t}+v t, t \geq 0\right)$ and $\left(W_{t}, t \geq 0\right)$, we obtain

$$
E\left[e^{-\theta \Sigma} g\left(X_{\Sigma}\right)\right]=\hat{E}_{0}\left[e^{-\left(\theta+\frac{v}{2}\right) T_{-a}} g\left(e^{-a}\right) e^{-v a} 1_{T_{-a}<T_{b}}\right]+\hat{E}_{0}\left[e^{-\left(\theta+\frac{v^{2}}{2}\right) T_{b}} g\left(e^{b}\right) e^{v b} 1_{T_{b}<T_{-A}}\right]
$$

Introducing $\frac{\mu^{2}}{2}=\theta+\frac{v^{2}}{2}$, it is straightforward to show (see e.g., Itô-Mc Kean (1965) or Revuz-Yor (1994)) that

$$
\hat{\mathrm{E}}_{\mathrm{o}}\left(\exp \left(-\frac{\mu^{2}}{2} \mathrm{~T}_{-\mathrm{a}}\right) ; \mathrm{T}_{-\mathrm{a}}<\mathrm{T}_{\mathrm{b}}\right)=\frac{\operatorname{sh} \mu \mathrm{b}}{\operatorname{sh}(\mu(\mathrm{a}+\mathrm{b}))}
$$

and

$$
\hat{\mathrm{E}}_{0}\left(\exp \left(-\frac{\mu^{2}}{2} \mathrm{~T}_{\mathrm{b}}\right) ; \mathrm{T}_{\mathrm{b}}<\mathrm{T}_{-\mathrm{a}}\right)=\frac{\operatorname{sh} \mu \mathrm{a}}{\operatorname{sh}(\mu(\mathrm{a}+\mathrm{b}))} .
$$

This is classically obtained by applying Doob's optional sampling theorem to exponential martingales of Brownian motion at $\mathrm{T}_{-a}$ and $\mathrm{T}_{\mathbf{b}}$. Then, using the strong Markov property (see Itô-Mc Kean for details), equation (6) becomes

$$
\Phi(\theta)=\frac{\operatorname{sh} \mu b}{\operatorname{sh}(\mu(a+b))} g\left(e^{-a}\right) e^{-v a}+\frac{\operatorname{sh} \mu a}{\operatorname{sh}(\mu(a+b))} g\left(e^{b}\right) e^{v b} .
$$


We need now to make explicit

$$
g\left(e^{z}\right)=V_{\theta}^{v} f\left(e^{z}\right) \stackrel{\text { def }}{=} \hat{E}_{z}\left(\int_{0}^{\infty} e^{-\theta u} f\left(e^{w_{u}+v u}\right) d u\right) .
$$

Using again the Cameron-Martin relationship, we obtain

$$
g\left(e^{z}\right)=e^{-v z} \hat{E}_{a}\left(\int_{0}^{\infty} \varphi_{v}\left(W_{u}\right) e^{-\frac{\mu^{2} u}{2}} d u\right),
$$

where $\varphi_{v}(y)=e^{v y} f\left(e^{y}\right)$ and $f(x)=(x-h)^{+}$.

The resolvent of Brownian motion (see e.g., Revuz-Yor, p. 93) is given for a general function $\varphi$ by

$$
\mathrm{U}_{\frac{\mu^{2}}{2}} \varphi(z)=\int_{0}^{+\infty} \mathrm{dt} \mathrm{e}^{-\frac{\mu^{2}}{2}} Y_{z}\left(\varphi\left(W_{\mathrm{t}}\right)\right)=\frac{1}{\mu} \int_{-\infty}^{+\infty} \mathrm{e}^{-\mu \mid z-y} \varphi(y) d y .
$$

In the case of the specific function $\varphi_{v}$ defined in (9), formula (10) gives

$$
\mathrm{U}_{\frac{\mu^{2}}{2}} \varphi_{v}(z)=\frac{1}{\mu} \int_{(\mathrm{rhh}}^{+\infty} d y\left(e^{-\mu|z-y|}\right)\left[e^{(v+1) y}-h e^{v y}\right]
$$

For notational simplicity, we introduce

$$
g_{1}\left(e^{z}\right) \equiv e^{v z} g\left(e^{z}\right)=U \frac{\mu^{2}}{2} \varphi_{v}(z)
$$

Relation (8) becomes

$$
\Phi(\theta)=\frac{\operatorname{sh}(\mu b)}{\operatorname{sh}[\mu(a+b)]} g_{1}\left(e^{-a}\right)+\frac{\operatorname{sh}(\mu a)}{\operatorname{sh}[\mu(a+b)]} g_{1}\left(e^{b}\right)
$$

where $g_{1}\left(e^{-a}\right)$ and $g_{1}\left(e^{b}\right)$ are shown, in Appendix 1 , to be respectively equal to 


$$
\mathrm{g}_{1}\left(\mathrm{e}^{-\mathrm{a}}\right)=\frac{h^{v+1-\mu} e^{-\mu \mathrm{a}}}{\mu(\mu-v)(\mu-v-1)}
$$

and

$$
g_{1}\left(e^{b}\right)=2\left\{\frac{e^{b(v+1)}}{\mu^{2}-(v+1)^{2}}-\frac{h e^{b v}}{\mu^{2}-v^{2}}\right\}+\frac{e^{-\mu b} h^{v+1+\mu}}{\mu(\mu+v)(\mu+v+1)}
$$

We recall that $\Phi$ depends on $\theta$ through $\mu=\sqrt{2 \theta+v^{2}}$ and that $\psi(\lambda)$, the quantity of interest, is related to $\Phi$ by the formula

$$
\psi(\lambda)=\frac{1}{\sigma^{2}} \Phi\left(\frac{\lambda}{\sigma^{2}}\right)
$$

Using this formula and denoting 1-1 $^{-1}$ for the inverse of the Laplace transform operator, it is immediate to see that

$$
\left\{\boldsymbol{x}^{-1} \Psi\right\}(\tau)=\left\{\mathbf{x}^{-1} \Phi\right\}\left(\sigma^{2} \tau\right)
$$

a result which may be substituted into equation (4) to give the price at time $t$ of the double-barrier call, namely

$$
C_{\mathrm{L}, \mathrm{U}}(t)=S(t)\left\{\mathrm{BS}(0,1, \sigma, \tau, h)-\mathrm{e}^{-\pi \tau}\left\{\mathbf{I}^{-1} \psi\right\}(\tau)\right\}
$$

where $B S(0,1, \sigma, \tau, h)$ is the Black and Scholes price of a standard call with maturity $\tau=T-t$, strike price $h=\frac{k}{S(t)}$, assumed to be written on an underlying asset $S$ such that $S(0)=1$.

\section{NUMERICAL APPLICATIONS}

We use formula (11) and the methodology developed in Geman-Eydeland (1995) in order to compute the price of a double-barrier option in three different settings; in all cases, we compare our results with the prices obtained through Monte-Carlo simulations and with the prices given in Kunitomo and Ikeda (1992). 
In the three tests, we use the values $S(0)=2, T=1$ year

\begin{tabular}{|c|c|c|c|}
\hline & $\begin{array}{l}\sigma=0.2 \\
r=0.02 \\
k=2, L=1.5, U=2.5\end{array}$ & $\begin{array}{l}\sigma=0.5 \\
r=0.05 \\
k=2, L=1.5, U=3\end{array}$ & $\begin{array}{l}\sigma=0.5 \\
r=0.05 \\
k=1.75, L=1, U=3\end{array}$ \\
\hline BS $(0,1, \sigma, \tau, \mathrm{h})$ & 0.0892 & 0.2179 & 0.27646 \\
\hline $\mathrm{e}^{-\mathrm{rT}}\left(\mathbf{X}^{-1} \Psi\right)(\mathrm{T})$ & 0.0687 & 0.2090 & 0.23838 \\
\hline G-Y price & 0.0411 & 0.0178 & 0.07615 \\
\hline K-I price & 0.041089 & 0.017856 & 0.076172 \\
\hline $\begin{array}{l}\text { Monte-Carlo price } \\
\text { (st. dev }=0.003 \text { ) }\end{array}$ & 0.0425 & 0.0191 & 0.0772 \\
\hline
\end{tabular}

The standard deviation is computed on a sample of 200 evaluations, each evaluation being performed on 5000 Monte-Carlo paths.

It is remarkable that in all cases, the price obtained through our method is extremely close to the Kunitomo-lkeda price. Moreover, in order for these two prices to lie within one standard deviation of the Monte-Carlo price, the Monte-Carlo simulations must be run using very small step sizes and many paths to make sure one barrier is not "hit but missed". The consequence is that the inversion of the Laplace transform requires an order of magnitude less operations than the Monte-Carlo simulations (e.g., it takes a fraction of a second to do it on a Sun Sparc 20 station). As a comparison, in the case of Asian options, Geman-Eydeland (1995) obtain a standard deviation as low as 0.001 for a sample of 50 evaluations, each of them being performed on 500 Monte-Carlo paths (and it is in the context of delta hedging that the inversion of the Laplace transform of the Asian option obtained in GemanYor (1993) proves definitely superior, both theoretically and computationally). Using Monte-Carlo methods, one faces a drastic change between the smoothing effect of the averaging feature of Asian options and the danger of hitting a barrier without knowing it in the case of double- 
knock options. It is not clear that all users of double-barrier options are fully aware of this problem.

A final manner to illustrate this point is to show that, as expected, the sensitivity of the option price to the step size in Monte-Carlo simulations becomes extremely high when the time remaining to maturity is short and the strike price close to one of the barriers.

We come back to the first column of the previous table with the same parameters except that $\tau=\mathrm{T}-\mathrm{t}=1$ month and $\mathrm{S}_{0}=2.4$.

For a step size equal to $1 / 365$ year, Monte-Carlo gives an option value equal to 0.1930 with a standard deviation $=0.073$; for a step size $\frac{1}{4 \times 365}$ year, the Monte-Carlo value becomes 0.1739 and the standard deviation 0.008 . Hence, a one day step gives a wrong result (the barrier may have been hit and "missed", and the option is overpriced).

Using the Geman-Yor approach, we obtain :

$\mathrm{BS}(0,1, \sigma, \tau, \mathrm{h})=0.1681 ; \mathrm{e}^{-\mathrm{rt}}\left(\mathrm{L}^{-1} \psi\right)(\tau)=0.23015$

G.Y price $=2.4 \times 0.1681-0.23015=0.17321$

\section{HEDGING DOUBLE-BARRIER OPTIONS}

Among the sensitivities of the option price to the different variables, the sensitivity to the underlying asset price is the first subject of concern for practitioners.

In order to compute the delta of the double-barrier option, we differentiate with respect to $S(t)$ the expression of $C_{L, U}(t)$ established in formula (12) and obtain

$$
\Delta=\frac{\partial C_{L, U}(t)}{\partial S(t)}=\left[B S(0,1, \sigma, \tau, h)-e^{-r \tau}\left\{L^{-1} \psi\right\}(\tau)\right]-S(t) e^{-r t} \frac{\partial}{\partial S(t)}\left\{L^{-1} \Psi\right\}(\tau)
$$

The quantity between brackets equals the call price divided by $S(t)$. We now need to find the derivative with respect to $S(t)$ of $\left\{\mathbb{Z}^{-1} \psi\right\}(\tau)$. 
To clearly exhibit the dependence in $S(t)$ of the function $\psi$, we write $S(t)=x$; $\psi_{x}(\lambda)=\frac{1}{\sigma^{2}} \Phi_{x}\left(\frac{\lambda}{\sigma^{2}}\right)$ and we express formula (11) in terms of $x$. Remembering that $m=e^{-a}$ and $M=e^{b}$, we obtain

$$
\begin{aligned}
\Phi_{x}(\theta) & =\frac{M^{\mu}-M^{-\mu}}{e^{\mu(a+b)}-e^{-\mu(a+b)}} g_{1}(m)+\frac{m^{-\mu}-m^{\mu}}{e^{\mu(a+b)}-e^{-\mu(a+b)}} g_{1}(M) \\
& =\frac{m^{\mu}\left(M^{2 \mu}-1\right)}{M^{2 \mu}-m^{2 \mu}} g_{1}(m)+\frac{M^{\mu}\left(1-m^{2 \mu}\right)}{M^{2 \mu}-m^{2 \mu}} g_{1}(M)
\end{aligned}
$$

where

$$
g_{1}(m)=\frac{m^{\mu} h^{v+1-\mu}}{\mu(\mu-v)(\mu-v-1)}
$$

and

$$
g_{1}(M)=2\left\{\frac{M^{v+1}}{\mu^{2}-(v+1)^{2}}-\frac{h M^{v}}{\mu^{2}-v^{2}}\right\}+\left(\frac{h}{M}\right)^{\mu} \frac{h^{v+1}}{\mu(\mu+v)(\mu+v+1)}
$$

Lastly, we need to write that $h=\frac{k}{x}, m=\frac{L}{x}, M=\frac{U}{x}$, which gives

$$
\begin{aligned}
\Phi_{x}(\theta) & =\frac{U^{2 \mu}-x^{2 \mu}}{U^{2 \mu}-L^{2 \mu}}\left(\frac{L}{x}\right)^{\mu}\left(\frac{k}{x}\right)^{v+1}\left(\frac{L}{k}\right)^{\mu} \frac{1}{\mu(\mu-v)(\mu-v-1)} \\
& +\frac{x^{2 \mu}-L^{2 \mu}}{U^{2 \mu}-L^{2 \mu}}\left(\frac{U}{x}\right)^{\mu} \frac{1}{x^{v+1}}\left[2\left\{\frac{U^{v+1}}{\mu^{2}-(v+1)^{2}}-\frac{k U^{v}}{\mu^{2}-v^{2}}\right\}+\left(\frac{k}{U}\right)^{\mu} \frac{k^{v+1}}{\mu(\mu+v)(\mu+v+1)}\right] .
\end{aligned}
$$

We can finally write

$$
\Phi_{x}(\theta)=\frac{U^{2 \mu}-x^{2 \mu}}{x^{\mu+v+1}} \alpha(L, U, k)+\frac{x^{2 \mu}-L^{2 \mu}}{x^{\mu+v+1}} \beta(L, U, k),
$$

where $\alpha(L, U, k)=\frac{L^{2 \mu} k^{v+1-\mu}}{U^{2 \mu}-L^{2 \mu}} \frac{1}{\mu(\mu-v)(\mu-v-1)}$,

$$
\beta(L, U, k)=\frac{1}{U^{2 \mu}-L^{2 \mu}}\left[2\left\{\frac{U^{\mu+v+1}}{\mu^{2}-(v+1)^{2}}-\frac{k U^{\mu+v}}{\mu^{2}-v^{2}}\right\}+\frac{k^{\mu+v+1}}{\mu(\mu+v)(\mu+v+1)}\right]
$$




$$
\begin{aligned}
& \Psi_{x}(\lambda)=\frac{1}{\sigma^{2}} \Phi_{x}\left(\frac{\lambda}{\sigma^{2}}\right), \\
& \frac{\partial}{\partial x}\left\{\mathcal{I}^{-1} \Psi_{x}\right\}(\tau)=\left\{\mathcal{I}^{-1} \frac{\partial \Psi_{x}}{\partial x}\right\}(\tau)=\left\{\mathcal{I}^{-1} \frac{\partial \Phi_{x}}{\partial x}\right\}\left(\sigma^{2} \tau\right) .
\end{aligned}
$$

We are consequently led to a problem of Laplace inversion nearly identical to the one encountered for the call price and involving $\frac{\partial \Phi_{x}}{\partial x}$ instead of $\Phi_{x^{\prime}}$ where

$$
\begin{aligned}
\frac{\partial \Phi_{x}}{\partial x}(\theta) & =\left[-(\mu-v-1) x^{\mu-v-2}-\frac{(\mu+v+1) U^{2 \mu}}{x^{\mu+v+2}}\right] \alpha(L, U, k) \\
& +\left[(\mu-v-1) x^{\mu-v-2}+\frac{(\mu+v+1) L^{2 \mu}}{x^{\mu+v+2}}\right] \beta(L, U, k) .
\end{aligned}
$$




\section{CONCLUSION}

From these results, we can easily derive the prices of double knock in options, i.e., options which pay like a vanilla call if the underlying spot price touches a lower or higher barrier prior to expiration but pay nothing (or a fixed rebate) if neither barrier is hit prior to expiration. In the same manner, double-barrier digital options, which pay a fixed amount if some reference asset price has remained within a band over the lifetime of the option, may be obtained as a special case of the valuation problem addressed in this paper. As in Geman-Yor (1993), this approach is satisfactory from a theoretical standpoint since the methodology is the same for the call price and for its delta. For practical purposes, the computational time of the two quantities is equally low and hedging - the ultimate concern of practitioners - is achieved with the same accuracy as pricing, which is rarely the case for path-dependent options. Moreover, the danger inherent-in Monte-Carlo methods applied to barrier and double-barrier options disappears in this approach. 


\section{BIBLIOGRAPHY}

BACHELIER L. (1941) "Probabilités des Oscillations Maxima", Note aux Comptes Rendus des Séances de l'Académie des Sciences

BLACK F. and M. SCHOLES, (1973) "The Pricing of Options and Corporate Liabilities", Journal of Political Economy, 81, 637-654.

CHESNEY M., JEANBLANC M. and M. YOR (1995) "Brownian Excursions and Parisian Barrier Options", To appear in Adv. in Appl. Proba.

DOOB J.L. (1949) "Heuristic Approach to the Kolmogorov-Smirnov Theorems" Annals of Mathematics Statistics 20, 393-403

FELLER W. (1970) "Introduction to Probability Theory and its Applications", Vol 2, 2nd edition, Wiley

FREEDMAN D. (1971) "Brownian Motion and Diffusion", Holden-Day

FU M., D. MADAN and T. WANG (1995) "Pricing Continuous Time Asian Options : A Comparison of Analytical and Monte Carlo Methods", Preprint, University of Maryland

GEMAN H. and A. EYDELAND (1995) "Domino Effect : Inverting the Laplace Transform", RISK, April

GEMAN H. and M. YOR, (1992) "Quelques relations entre processus de Bessel, Options Asiatiques et Fonctions Confluentes Hypergéométriques", Notes aux Comptes Rendus de l'Academie des Sciences, Sér. I., 471-474

GEMAN H. and M. YOR, (1993) "Bessel Processes, Asian Options and Perpetuities", Mathematical Finance, 3, N4, 349-375

GOLDMAN, M., H. SOSIN and M. GATTO (1979) "Path Dependent Options : Buy at the Low, Sell at the High", Journal of Finance, 34, 111-127

HARRISON J.M. and D. KREPS (1979) "Martingales and Arbitrage in Multiperiod Securities Markets", Journal of Economic Theory 20, 381-408

HARRISON J.M. and S. R. PLISKA (1981) "Martingales and Stochastic Integrals in the Theory of Continuous Trading", Stoch. Proc. Appl, 11, 215260 
KUNITOMO N. and M. IKEDA (1992) "Pricing Options with Curved Boundaries", Mathematical Finance, Vol. 2, N4, 275-2

ITÔ K. and H.P. Mc KEAN (1965) "Diffusion Processes and Their Sample Paths", Springer

MERTON R.C. (1973) "Theory of Rational Option Pricing", Bell. J. Econ. Manag. Sci., Vol 4, p. 141-183

REVUZ D. and M. YOR (1994) "Continuous Martingales and Brownian Motion", 2nd edition, Springer

SHORACK G.R. and J.A. WELLNER (1986) "Empirical Processes with Applications to Statistics", Wiley

TEUNEN M. and GOOVAERTS M. (1994) "Double Boundary Crossing Result for the Brownian Motion", Scand. Act. J. ,Vol 2 


\section{APPENDIX 1}

\section{Computation of $\frac{\mathrm{U}^{2}}{2} \varphi(z)$}

We recall that $\mu=\sqrt{2 \theta+v^{2}}$, hence $\mu>v$. We are looking for a simple expression of $U \frac{\mu^{2}}{2} \varphi(z)$. We know that

$$
\mathrm{U} \frac{\mu^{2}}{2} \varphi(z)=\frac{1}{\mu} \int_{\ell \mathrm{nh}}^{\infty} \mathrm{e}^{-\mu|z-y|}\left[e^{(v+1) y}-\mathrm{h} \mathrm{e}^{\mathrm{v} y}\right] \mathrm{dy} \stackrel{\operatorname{def}}{=} \mathrm{H}_{\mu}^{z}(\operatorname{lnh}, v+1)-\mathrm{h} \mathrm{H}_{\mu}^{z}(\operatorname{lnh}, v),
$$

where

$$
\mathrm{H}_{\mu}^{z}(\operatorname{lnh}, \mathrm{m})=\frac{1}{\mu} \int_{(\mathrm{nh})}^{\infty} \mathrm{e}^{-\mu|z-y|} e^{\mathrm{my}} \mathrm{dy}
$$

In the case of $g_{1}\left(e^{-a}\right) \equiv U \frac{\mu^{2}}{2} \varphi(-a)$, the integrals

$$
\mathrm{K}_{\mu}^{z}(\operatorname{lnh}, \mathrm{m}) \equiv \int_{(\mathrm{nh}}^{+\infty} \mathrm{e}^{-\mu|z-y|+m y} d y \quad(\mathrm{~m}=\mathrm{v} \text { or } \mathrm{v}+1)
$$

do not need to be split since $z=-a$ is smaller than $\ell$ nh and one easily obtains

$$
g_{1}\left(e^{-a}\right)=\frac{e^{-\mu(a+(n h)} e^{(v+1)(n h}}{\mu(\mu-v-1)}-h \frac{e^{-\mu(a+/ n h)} e^{v / n h}}{\mu(\mu-v)}=\frac{h^{v+1-\mu} e^{-\mu a}}{\mu(\mu-v)(\mu-v-1)}
$$

For the computation of $g_{1}\left(e^{b}\right)=U_{\frac{\mu^{2}}{2}} \varphi(b)$, both integrals $\int_{i n h}^{+\infty} e^{-\mu|z-y|+m y} d y$ $(m=v$ and $v+1)$ have to be split since $z=b$ is bigger than $\ell n h$; this leads to the following expression :

$$
\begin{aligned}
g_{1}\left(e^{b}\right) & =\frac{1}{\mu}\left[\frac{e^{b(v+1)}}{\mu+v+1}+\frac{e^{b(v+1)}}{\mu-v-1}-\frac{e^{\mu(\text { (nh-b)+(v+1)/nh }}}{\mu+v+1}\right]-\frac{h}{\mu}\left[\frac{e^{b v}}{\mu+v}+\frac{e^{b v}}{\mu-v}-\frac{e^{\mu(\text { (nh }-b)+v(n h}}{\mu+v}\right] \\
& =\frac{1}{\mu}\left\{e^{b(v+1)} \frac{2 \mu}{\mu^{2}-(v+1)^{2}}-e^{\mu(\ell n h-b)+v \ell n h} \frac{h}{\mu+v+1}\right\} \\
& -\frac{h}{\mu}\left\{e^{v b} \frac{2 \mu}{\mu^{2}-v^{2}}-\frac{e^{\mu(\ell n h-b)+v \ell n h}}{\mu+v}\right\} \\
& =2\left\{\frac{e^{b(v+1)}}{\mu^{2}-(v+1)^{2}}-\frac{h e^{b v}}{\mu^{2}-v^{2}}\right\}+\frac{e^{-\mu b} h^{\mu+v+1}}{\mu(v+1)(\mu+v+1)} .
\end{aligned}
$$




\section{APPENDIX 2}

Why are the laws of functionals of $X_{t}=\sigma W_{t}+v t$ (Brownian motion with constant volatility $\sigma>0$ and drift $v$ ) such as $M_{t}=\sup _{u \leq t} X_{u}$ or $I_{t}=-\inf _{u \leq t} X_{u}$ simpler when the fixed time $t$ is replaced by an exponential time $\tau$ independent of W ? A first explanation stems from the lack of memory of the exponential variable; but a deeper answer may be given in terms of excursion theory, which implies for instance that $M_{\tau}-X_{\tau}$ and $M_{\tau}$ are independent. This property is also well-known to be a consequence of the Wiener-Hopf factorization, which is referred to by Rogers and Satchell (1991) when looking at high, low and closing stock prices. However, Rogers and Satchell observed in their paper that cross-moments such as $E\left[M_{\tau} I_{\tau}\left(M_{\tau}+I_{\tau}\right)\left(X_{\tau}+I_{\tau}\right)\right]$ are hard to compute in the case of a nonzero drift $v$. We show below that the distribution of the triple $\left(\mathrm{X}_{\tau}, \mathrm{M}_{\tau}, \mathrm{I}_{\tau}\right)$ - a quantity of great interest throughout our paper - has a fairly simple expression; its use may in particular allow to compute, at the cost of a multiple integral, the above mentioned expectation.

Let us denote $\tau_{\theta}$ an exponential time independent of $X$, with parameter $\frac{\theta^{2}}{2}$.

Then, we have the formula

$$
\mathrm{P}\left(\mathrm{M}_{\tau_{\mathrm{\theta}}} \leq \mathrm{a}, \mathrm{I}_{\tau_{\mathrm{\theta}}} \leq \mathrm{b}, \mathrm{X}_{\tau_{\mathrm{\theta}}} \in \mathrm{dz}\right)=\left(\frac{\theta^{2}}{\mu \sigma}\right) \varphi\left(\frac{\mathrm{a} \mu}{\sigma}, \frac{\mathrm{b} \mu}{\sigma}, \frac{z \mu}{\sigma}\right) \exp \left(\frac{v \mathrm{z}}{\sigma^{2}}\right) \mathrm{d} \mathrm{z}
$$

where $\mu=\sqrt{\theta^{2}+\left(v^{2} / \sigma^{2}\right)}$ and

$\varphi(a, b, x)=\frac{\sinh (|x|)}{\operatorname{coth} a+\operatorname{coth} b}\left\{\begin{array}{l}\operatorname{coth} x-\operatorname{coth} a, \text { if } 0<x<a \\ \operatorname{coth}|x|-\operatorname{coth} b, \text { if }-b<x<0\end{array}\right.$

Proof

1. The formula in the "reduced case" $\theta=1, \sigma=1, \nu=0$ is found in Pitman-Yor (1992) and has been obtained using excursion theory.

2. We now show how to obtain the general formula, using essentially the Cameron-Martin relation between Brownian motions with zero and nonzero drift, and the scaling property of Brownian motion. 
(2i) We first show the formula in the case $\sigma=1$.

We denote $W_{t}^{(v)}=W_{t}+v t, M_{t}^{(v)}=\sup _{s s t} W_{s}^{(v)}, I_{t}^{(v)}=-\inf _{s \leq t} W_{s}^{(v)}$

We have, for any measurable $f: R^{+} \times R^{+} \times R->R$

$$
\begin{aligned}
& \gamma_{f} \equiv E\left[f\left(M_{\tau_{\theta}}^{(v)}, I_{\tau_{\theta}}^{(v)}, W_{\tau_{\theta}}^{(v)}\right)\right]=E\left[f\left(M_{\tau_{\theta}}, I_{\tau_{\theta}}, W_{\tau_{\theta}}\right) \exp \left\{v W_{\tau_{\theta}}-\frac{v^{2}}{2} \tau_{\theta}\right\}\right] \\
& =\frac{\theta^{2}}{2} \int_{0}^{\infty} d t \exp \left\{-\left(\frac{v^{2}+\theta^{2}}{2}\right) t\right\} E\left[f\left(M_{t}, I_{t}, W_{t}\right) \exp \left(v W_{t}\right)\right] \\
& =\frac{\theta^{2}}{\mu^{2}} E\left[f\left(M_{\tau_{\mu}}, I_{\tau_{\mu}}, W_{\tau_{\mu}}\right) \exp \left(v W_{\tau_{\mu}}\right)\right]
\end{aligned}
$$

Thanks to the scaling with respect to $\tau_{\mu}$, i.e., $\tau_{\mu}^{\text {law }}=\frac{1}{\mu^{2}} \tau_{1}$, we obtain

$$
\gamma_{f}=\frac{\theta^{2}}{\mu^{2}} E\left[f\left(\frac{1}{\mu} M_{\tau_{1}}, \frac{1}{\mu} I_{\tau_{1}}, \frac{1}{\mu} W_{\tau_{1}}\right) \exp \left(\frac{v}{\mu} W_{\tau_{1}}\right)\right] .
$$

We now use the Pitman-Yor result in the "reduced case" and (1) in order to derive

$$
\begin{aligned}
& E\left[M_{\tau_{\theta}}^{(v)} \leq a, I_{\tau_{\tau_{0}}}^{(v)} \leq b ; f\left(W_{\tau_{\theta}}^{(v)}\right)\right]=\frac{\theta^{2}}{\mu^{2}} P\left(M_{\tau_{1}} \leq a \mu, I_{\tau_{1}} \leq b \mu ; f\left(\frac{1}{\mu} W_{\tau_{1}}\right) \exp \left(\frac{\nu}{\mu} W_{\tau_{1}}\right)\right) \\
& =\frac{\theta^{2}}{\mu^{2}} \int d x \varphi(a \mu, b \mu, x) f\left(\frac{x}{\mu}\right) \exp \left(\frac{v}{\mu} x\right) \\
& =\frac{\theta^{2}}{\mu} \int d y \varphi(a \mu, b \mu, \mu y) f(y) \exp (v y)
\end{aligned}
$$

which gives the result in the case $\sigma=1$.

(2ii) To get the general formula, we write

$$
\sigma W_{t}+v t \equiv \sigma\left(W_{t}+\frac{v}{\sigma} t\right)
$$


We denote $M_{(1)}, I_{(1)}, W_{(1)}$ the random quantities in (1) where $v$ is replaced by $\frac{v}{\sigma}$

$$
\begin{aligned}
& \mathrm{E}\left[\sigma \mathrm{M}_{(1)} \leq \mathrm{a} ; \sigma \mathrm{I}_{(1)} \leq \mathrm{b} ; \mathrm{f}\left(\sigma \mathrm{W}_{(1)}\right)\right]=\mathrm{E}\left[\mathrm{M}_{(1)} \leq \frac{\mathrm{a}}{\sigma} ; \mathrm{I}_{(1)} \leq \frac{\mathrm{b}}{\sigma} ; \mathrm{f}\left(\sigma \mathrm{W}_{(1)}\right)\right] \\
& =\int \mathrm{dy} \psi\left(\frac{\mathrm{a}}{\sigma}, \frac{\mathrm{b}}{\sigma}, \mathrm{y}\right) \mathrm{f}(\sigma \mathrm{\sigma})=\int \frac{\mathrm{dz}}{\sigma} \psi\left(\frac{\mathrm{a}}{\sigma}, \frac{\mathrm{b}}{\sigma}, \frac{\mathrm{z}}{\sigma}\right) \mathrm{f}(\mathrm{z})
\end{aligned}
$$

where $\psi$ refers to the density function obtained in (2) but with $v$ replaced by $\frac{v}{\sigma}$.

Hence our final quantity is

$$
\frac{1}{\sigma} \psi\left(\frac{\mathrm{a}}{\sigma}, \frac{\mathrm{b}}{\sigma}, \frac{\mathrm{z}}{\sigma}\right) \equiv\left(\frac{\theta^{2}}{\mu \sigma}\right) \varphi\left(\frac{\mathrm{a} \mu}{\sigma}, \frac{\mathrm{b} \mu}{\sigma}, \frac{\mathrm{z} \mu}{\sigma}\right) \exp \left(\frac{\mathrm{vz}}{\sigma^{2}}\right)
$$

\section{REFERENCES}

PITMAN. J and M. YOR (1992) : "The laws of Homogeneous Functionals of Brownian Motion and Related Processes", Preprint, University of Berkeley

ROGERS L.C.G. and S.E. SATCHELL (1991) "Estimating Variance from High, Low and Closing Prices", Annals of Applied Probability, Vol 1., N4, 504-512 